\title{
Higher-order finite volume method with semi-Lagrangian scheme for one-dimensional conservation laws
}

\author{
Lang $\mathrm{Wu}^{1}$, Songsong $\mathrm{Li}^{1,2}$ and Boying $\mathrm{Wu}^{1 *}$
}

\section{"Correspondence:}

mathwby@163.com

'Department of Mathematics,

Harbin Institute of Technology,

No. 92, West Da-Zhi Street, Harbin,

150001, China

Full list of author information is

available at the end of the article

\begin{abstract}
In this paper, a high-order, semi-Lagrangian finite volume (SL-FV) method based on the WENO approach is proposed in order to manage one-dimensional conservation laws. The proposed method successfully integrates WENO reconstructions and the semi-Lagrangian method. More specifically, the Taylor expansion of time is used to approximate the time integration, deployed to boost temporal accuracy. Next, characteristic curves are applied to replace the time level by points in the semi-Lagrangian method. The value of these points can then be reconstructed by WENO schemes to increase their accuracy in space. Both high-order accuracies in space and time, respectively, are achieved. Moreover, computational experiments allow for a weaker CFL condition, provided in detail to validate the performance of the proposed SL-FV-based WENO method.
\end{abstract}

Keywords: semi-Lagrangian method; WENO reconstructions; Taylor expansion; Euler system

\section{Introduction}

One-dimensional conservation laws are considered in this paper, detailed in the following equation:

$$
\begin{aligned}
& v_{t}+g(v)_{x}=0, \\
& v(x, 0)=v_{0}(x) .
\end{aligned}
$$

For problems such as these, this paper develops a series of semi-Lagrangian finite volume (SL-FV) WENO schemes which allow for weaker CFL conditions, while maintaining highorder precision and preventing shock transitions near discontinuities. The SL approach is based on the compound mode of the Lagrangian and Eulerian approaches, and thus possesses inherent advantages of the two schemes. Lagrangian and semi-Lagrangian insights are already quite widely applied to computational fluid dynamics and astrophysics [1-4].

The Lagrangian-Eulerian formulation with moving grids was applied to unsteady, compressible Euler equations, under a conservative scheme [5]. The Eulerian method was able to manage the strongly nonlinear processes without introducing additional complexity. The Lagrangian method was developed in FV, where the scheme essentially combined with the non-oscillatory (ENO) method to solve the Euler equations [6]. The scheme 
obtained high-order precision with Cartesian and cylindrical coordinates. Liu et al. developed a Lagrangian method with a Taylor expansion in time, called the Lax-Wendroff method, developed in order to solve compressible Euler equations and fulfill planned accuracy in compact spatial stencils [7]. In another study [8, 9], the Lagrangian scheme was used to determine a solution for compressible fluid flows in cylindrical coordinates, proven to maintain symmetry and conservation characteristics. As for columniform problems, by way of obtaining a method which preserves circular harmony and conservative characteristics, the momentum equation was discretized alongside the local polar and angular directions.

In yet another relevant study [10], Cheng and Knorr proposed an SL method, to considerable interest of computational scientists. According to the property of characteristic curves, the SL method calculated the value of points which end at the grid point backward in time. Over the last few years, a semi-Lagrangian methodology has been explored at length for its ability to solve the Vlasov equation [11,12] and transport scheme [1315]. The semi-Lagrangian method can be divided into two categories: the backward semiLagrangian (BSL) method, and forward semi-Lagrangian (FSL) method. The BSL method utilizes the point value with $t^{n+1}$, where the value point $t^{n}$ must be identified; the FSL method functions in a manner exactly opposite. Both semi-Lagrangian methods utilized Lagrange reconstruction, spline reconstruction, and Hermite reconstruction to compute their characteristic variables [11].

The semi-Lagrangian scheme maintains uniform high-order precision and non-oscillatory shock transitions near discontinuities, which simultaneously allowed weaker CFL conditions to ensure computational efficiency. Semi-Lagrangian methods have been applied more recently to FV structures, and to discontinuous Galerkin and FD schemes [16-19]. Specifically, in one recent study [18, 19], Qiu and Shu developed an SL finite difference method using WENO approaches to approximate the advection equation and the Vlasov equation. The FD WENO approach used point values instead of cell averages for the Strang split scheme. A positivity preservation of the semi-Lagrangian discontinuous Galerkin method was also proposed [20], which demonstrated overall consistency and preciseness. Another study [16] proposed a maximum principle preserving the semiLagrangian discontinuous Galerkin method, and it further developed a positivity preserving limiter.

High-order SL-FV approaches are created to approximate scalars and Euler equations by combining the advantages of WENO reconstructions [21-25] and the semi-Lagrangian method. To this effect, this type of approach reaches high-order accuracies in both space and time. The objective of employing the Taylor expansion method for time is a convenient procedure that manages integration in time and increases temporal accuracy. In another relevant study [11], Crouseilles et al. presented several methods to approximate the solution of characteristics curves, but we adopt a RK method to accurately tract the characteristics curves. So this study utilizes WENO procedures to achieve a uniform high-order precision.

The remainder of this paper is organized as follows. Section 2 reviews FV WENO reconstruction for scalar cases. Section 3 describes the SL-FV scheme and offers WENO reconstructions for both scalar and hyperbolic conservation law systems. In Section 4, experimental results confirming the efficiency of the approaches are provided. Section 5 summarizes and concludes the study, and it offers potential future research direction. 


\section{Review of FV method for scalar conservation laws}

We shall follow $[21,22]$ to review the FV WENO schemes for the scalar equation formulated by

$$
\begin{aligned}
& v_{t}+g(v)_{x}=0, \\
& v(x, 0)=v_{0}(x) .
\end{aligned}
$$

This has a conservation law form. The computational domain of this problem which is considered in this paper is $[a, b]$. We would like to adopt the specific cell to involve $[a, b]$, which is composed of cells $I_{j}=\left[x_{j-\frac{1}{2}}, x_{j+\frac{1}{2}}\right]$, for $j=1, \ldots, N$, where

$$
a=x_{\frac{1}{2}}<x_{\frac{3}{2}}<\cdots<x_{N+\frac{1}{2}}=b
$$

The mesh center is denoted by $x_{j}=\frac{1}{2}\left(x_{j-\frac{1}{2}}+x_{j+\frac{1}{2}}\right)$ and the mesh length is denoted by $\Delta x_{j}=$ $x_{j+\frac{1}{2}}-x_{j-\frac{1}{2}}$. The values of $v$ at the discontinuity point $x_{j+\frac{1}{2}}$ are indicated by $v_{j+\frac{1}{2}}^{-}$and $v_{j+\frac{1}{2}}^{+}$. Throughout the paper, we use the function $\bar{v}_{j}(t)$ to indicate the cell average of $v(x)$, namely, $\bar{v}_{j}(t)=\frac{1}{\Delta x_{j}} \int_{I_{j}} v(x, t) d x$ over the cell $I_{j}$. Let us now resolve an integrated version of (2.1) to arrive at the following finite volume scheme:

$$
\frac{d}{d t} \bar{v}_{j}(t)=-\frac{1}{\Delta x_{j}}\left(\hat{g}_{j+\frac{1}{2}}-\hat{g}_{j-\frac{1}{2}}\right)
$$

for $j=1, \ldots, N$, where the numerical flux is $\hat{g}_{j+\frac{1}{2}}=\hat{g}\left(v_{j+\frac{1}{2}}^{-}, v_{j+\frac{1}{2}}^{+}\right)$, which is usually chosen as monotone fluxes. The approximations $v_{j+\frac{1}{2}}^{-}$and $v_{j+\frac{1}{2}}^{+}$are reconstructed by the WENO methodology described as follows.

For high-order WENO reconstructions, say, of $2 k+1$ th-order of accuracy, we first need to recognize $k+1$ small templates $S_{\tau}, \tau=0, \ldots, k$, the cell $I_{j}$ is part of each of them. Generality, here we make $S_{\tau}=\bigcup_{l=0}^{k} I_{j+\tau-l}$. We then utilize $S=\bigcup_{\tau=0}^{k} S_{\tau}$ to describe the larger template, which consists of all the stencils from the $k+1$ smaller templates. We built a $k$ th degree polynomial reconstruction in each of the cells $S_{\tau}, \tau=0, \ldots, k$, which is indicated by $H_{\tau}(x)$. Namely, the cell average of $H_{\tau}(x)$ in the template $S_{\tau}$ is in accord with the given cell average of $v$. Analogously, associated with the larger stencil $S$, we also find a (2k)th-order polynomial, $G(x)$. The details of reconstructing the polynomials $H_{\tau}(x)$ and $G(x)$ can be learned, for example, from [26].

From the above discussions, we have

$$
\begin{aligned}
& \bar{v}_{j+\tau-l}=\frac{1}{\Delta x_{j+\tau-l}} \int_{I_{j+\tau-l}} H_{\tau}(x) d x, \quad \tau, l=0, \ldots, k, \\
& \bar{v}_{j+l}=\frac{1}{\Delta x_{j+l}} \int_{I_{j+l}} G(x) d x, \quad l=-k, \ldots, k .
\end{aligned}
$$

Further, the so-called linear weighs, denoted by $c_{0}, c_{1}, \ldots, c_{k}$, can be determined if we evaluate the reconstructed polynomials $H_{\tau}(x)$ and $G(x)$ at a specific point, say, $x_{j+\frac{1}{2}}$, which satisfy

$$
G\left(x_{j+\frac{1}{2}}\right)=\sum_{\tau=0}^{k} c_{\tau} H_{\tau}\left(x_{j+\frac{1}{2}}\right) .
$$


It is worthwhile to note that we easily can utilize $c_{\tau}$ and a linear combination, (2.3), and we would get an approach that will be oscillatory at the discontinuities. To handle this problem, we replace $c_{\tau}$ in (2.3) with nonlinear weights $\omega_{\tau}$, which result in an approach is high-order precision and non-oscillatory at the discontinuities. In fact, this is typically accomplished with the help of the smoothness indicators, denoted by $I Y_{\tau}$, which estimate the smooth properties of $H_{\tau}(x)$ in each cell $I_{j}$ for each small stencil $S_{\tau}$. As in $[22,26]$, the smoothness indicators have the form

$$
I Y_{\tau}=\sum_{l=1}^{k} \int_{I_{j}} \Delta x_{j}^{2 l-1}\left(\frac{\partial^{l} H_{\tau}(x)}{\partial x^{l}}\right)^{2} d x
$$

The nonlinear weights are calculated in the following way:

$$
\omega_{\tau}=\frac{\delta_{\tau}}{\sum_{s=0}^{k} \delta_{s}}, \quad \delta_{\tau}=\frac{c_{\tau}}{\left(\varepsilon+I Y_{\tau}\right)^{2}}, \quad \tau=0, \ldots, k
$$

in which the $\varepsilon$ is positive and minute enough to keep the denominator away from 0 . So the $\varepsilon$ is selected as $10^{-6}$ for my simulations. The scheme uses the nonlinear weights is steadily. So far, the WENO construction is computed by the form

$$
v_{j+\frac{1}{2}}^{-}=\sum_{\tau=0}^{k} \omega_{\tau} H_{\tau}\left(x_{j+\frac{1}{2}}\right)
$$

The approximate value $v_{j-\frac{1}{2}}^{+}$has mirror symmetry in the above procedure.

The WENO algorithm is now well defined.

\section{High-order semi-Lagrangian FV method for one-dimensional problems}

In the part, the detailed procedures of the SL-FV WENO 3 and WENO 5 approaches are designed for scalar and Euler equations.

\subsection{SL-FV method for the scalar problem}

Now, we begin to propose the SL-FV scheme in this section. Let us start by an integrated version of (2.1) in space and time to get the SL-FV scheme

$$
\bar{v}_{j}^{n+1}=\bar{v}_{j}^{n}-\frac{1}{\Delta x_{j}} \int_{t^{n}}^{t^{n+1}}\left(\hat{g}_{j+\frac{1}{2}}-\hat{g}_{j-\frac{1}{2}}\right) d t
$$

In this article, the Taylor expansion is utilized to estimate the above equation. Through the homologous derivation, we have the following form:

$$
\int_{t^{n}}^{t^{n+1}} \hat{g}_{j+\frac{1}{2}} d t=\sum_{l=0}^{m-1} D_{l}\left(v\left(x_{j+\frac{1}{2}}, t^{n+\frac{1}{2}}\right)\right) B(l)+o\left(\Delta t^{(m+1)}\right) .
$$

Here, $l$ is even number, we define $D_{l}(v(x, t))=\frac{\partial^{l} \hat{g}(v(x, t))}{\partial t^{l}}$ and $B(l)=\frac{1}{(l+1) !} \frac{1}{2^{l}} \Delta t^{(l+1)}$. In the trajectories of the characteristic lines, we can find the point $x_{0}^{(n)}$, the value $v\left(x_{0}^{(n)}, t^{n}\right)$ is equal 
to $v(x, t)$, when it is located at the point $x_{j+\frac{1}{2}}$ on the time level $t=t^{n+\frac{1}{2}}$. This is a most important thing in building the SL-FV method, and the point $x_{0}^{(n)}$ can be reached by

$$
\left\{\begin{array}{l}
x_{j+\frac{1}{2}}^{(1)}=x_{j+\frac{1}{2}}-\frac{1}{8} \Delta \operatorname{tg}^{\prime}\left(x_{j+\frac{1}{2}}\right), \\
x_{j+\frac{1}{2}}^{(2)}=x_{j+\frac{1}{2}}-\frac{1}{4} \Delta \operatorname{tg}^{\prime}\left(x_{j+\frac{1}{2}}^{(1)}\right), \\
x_{j+\frac{1}{2}}^{(3)}=x_{j+\frac{1}{2}}-\frac{1}{2} \Delta t\left(g^{\prime}\left(x_{j+\frac{1}{2}}\right)-2 g^{\prime}\left(x_{j+\frac{1}{2}}^{(1)}\right)+2 g^{\prime}\left(x_{j+\frac{1}{2}}^{(2)}\right)\right) \\
x_{0}^{(n)}=x_{j+\frac{1}{2}}-\frac{1}{12} \Delta t\left(g^{\prime}\left(x_{j+\frac{1}{2}}\right)+4 g^{\prime}\left(x_{j+\frac{1}{2}}^{(2)}\right)+g^{\prime}\left(x_{j+\frac{1}{2}}^{(3)}\right)\right)
\end{array}\right.
$$

Taking the outcome, $x_{0}^{(n)}$, in (3.1), which is determined by the characteristic lines and through the corresponding transformation, we then obtain the SL-FV method,

$$
\bar{v}_{j}^{n+1}=\bar{v}_{j}^{n}-\frac{1}{\Delta x_{j}} \sum_{l=0}^{m-1} B(l)\left(D_{l}\left(v\left(x_{0}^{(n)}, t^{n}\right)\right)-D_{l}\left(v\left(x_{j-\frac{1}{2}}^{(n)}, t^{n}\right)\right)\right) .
$$

Up to now, one can see that all the values, $v\left(x_{0}^{(n)}, t^{n}\right)$ are reconstructed in the next section. Now, we begin to introduce the WENO reconstructions.

\subsection{WENO reconstructions for the SL-FV scheme of the scalar conservation laws}

As everyone knows, the WENO schemes can achieve high-order precision and capture shock at the discontinuities. Next, we shall present the WENO reconstructions of third order and fifth order for the SL-FV scheme, respectively.

\subsection{1 $g^{\prime}(x, t) \geq 0$ in the SL-FV scheme}

Third-order WENO reconstruction In the interest of acquiring the approximations $v$ at the point $x_{0}^{(n)}$ with time level $t^{n}$, for example, $\left(v_{0}^{(n)}\right)^{-}$, we denote $\rho_{j}(x)=\frac{x_{j+\frac{1}{2}}^{-x}}{\Delta x_{j}}$ and reproduce the multinomial $H_{\tau}(x)$,

$$
H_{\tau}(x)=\operatorname{COE}_{1}\left(\frac{1}{2}-\rho_{j}(x)\right)+\operatorname{COE}_{2} \quad(\tau=0,1)
$$

associated with smaller templates $S_{\tau}=\bigcup_{l=0}^{1} I_{j+\tau-l}, \tau=0,1$, to build the scheme

$$
\bar{v}_{j+\tau-l}=\frac{1}{\Delta x_{j+\tau-l}} \int_{I_{j+\tau-l}}\left(\operatorname{COE}_{1}\left(\frac{1}{2}-\rho_{j}(x)\right)+C O E_{2}\right) d x, \quad \tau, l=0,1,
$$

and we acquire the polynomials

$$
\begin{aligned}
& H_{0}\left(x_{0}^{(n)}\right)=\left(-\frac{1}{2}+\rho_{j}\left(x_{0}^{(n)}\right)\right) \bar{v}_{j-1}^{n}+\left(\frac{3}{2}-\rho_{j}\left(x_{0}^{(n)}\right)\right) \bar{v}_{j}^{n}, \\
& H_{1}\left(x_{0}^{(n)}\right)=\left(\frac{1}{2}+\rho_{j}\left(x_{0}^{(n)}\right)\right) \bar{v}_{j}^{n}+\left(\frac{1}{2}-\rho_{j}\left(x_{0}^{(n)}\right)\right) \bar{v}_{j+1}^{n},
\end{aligned}
$$

and build a multinomial $G(x)$,

$$
G(x)=\operatorname{COE}_{1}\left(\frac{1}{2}-\rho_{j}(x)\right)^{2}+\operatorname{COE}_{2}\left(\frac{1}{2}-\rho_{j}(x)\right)+\operatorname{COE}_{3}
$$


which is associated with the larger template $S=\bigcup_{\tau=0}^{1} S_{\tau}$, to build the scheme

$$
\bar{v}_{j+l}=\frac{1}{\Delta x_{j+l}} \int_{I_{j+l}}\left(\operatorname{COE}_{1}\left(\frac{1}{2}-\rho_{j}(x)\right)^{2}+\operatorname{COE}_{2}\left(\frac{1}{2}-\rho_{j}(x)\right)+C O E_{3}\right) d x, \quad l=-1,0,1,
$$

we acquire the polynomial

$$
\begin{aligned}
G\left(x_{0}^{(n)}\right)= & \left(-\frac{1}{6}+\frac{\rho_{j}\left(x_{0}^{(n)}\right)^{2}}{2}\right) \bar{v}_{j-1}^{n}+\left(\frac{5}{6}+\rho_{j}\left(x_{0}^{(n)}\right)-\rho_{j}\left(x_{0}^{(n)}\right)^{2}\right) \bar{v}_{j}^{n} \\
& +\left(\frac{1}{3}-\rho_{j}\left(x_{0}^{(n)}\right)+\frac{\rho_{j}\left(x_{0}^{(n)}\right)^{2}}{2}\right) \bar{v}_{j+1}^{n},
\end{aligned}
$$

the linear weights can be found at the point $x_{0}^{(n)}$, expressed by $c_{0}, c_{1}$,

$$
c_{0}=\frac{-\frac{1}{6}+\frac{\rho_{j}\left(x_{0}^{(n)}\right)^{2}}{2}}{-\frac{1}{2}+\rho_{j}\left(x_{0}^{(n)}\right)}, \quad c_{1}=\frac{\frac{1}{3}-\rho_{j}\left(x_{0}^{(n)}\right)+\frac{\rho_{j}\left(x_{0}^{(n)}\right)^{2}}{2}}{\frac{1}{2}-\rho_{j}\left(x_{0}^{(n)}\right)} .
$$

We chose the following form as the smoothness indicators in the stencils $S_{0}, S_{1}$ :

$$
I Y_{0}=\left(\bar{v}_{j}^{n}-\bar{v}_{j-1}^{n}\right)^{2}, \quad I Y_{1}=\left(\bar{v}_{j+1}^{n}-\bar{v}_{j}^{n}\right)^{2} .
$$

The corresponding nonlinear weights can be written as

$$
\omega_{\tau}=\frac{\delta_{\tau}}{\sum_{s=0}^{1} \delta_{s}}, \quad \delta_{\tau}=\frac{c_{\tau}}{\left(\varepsilon+I Y_{\tau}\right)^{2}}, \quad \tau=0,1
$$

Here $\varepsilon>0$ is to keep the denominator away from 0 , and the $\varepsilon$ is utilized as $10^{-6}$ for the simulations.

Thus, we have the reconstructed point value of $v$ at $x_{0}^{(n)}$ at time level $t^{n}$, which can be indicated by

$$
\left(v_{0}^{(n)}\right)^{-}=\sum_{\tau=0}^{1} \omega_{\tau} H_{\tau}\left(x_{0}^{(n)}\right) .
$$

We remark that when the CFL number is large enough to make $x_{j+\frac{1}{2}}-x_{0}^{(n)}>\Delta x_{j}$, the point $x_{0}^{(n)}$ would be located at the corresponding meshes. So we build the WENO reconstruction to the corresponding template around $x_{0}^{(n)}$.

Fifth-order WENO reconstruction We reconstruct the multinomials $H_{\tau}(x)$, which are associated with three smaller templates $S_{\tau}=\bigcup_{l=0}^{2} I_{j+\tau-l}, \tau=0,1,2$, to design the scheme

$$
\bar{v}_{j+\tau-l}=\frac{1}{\Delta x_{j+\tau-l}} \int_{I_{j+\tau-l}} H_{\tau}(x) d x \quad \tau, l=0,1,2,
$$

and design a multinomial $G(x)$, which is associated with the larger template $S=\bigcup_{\tau=0}^{2} S_{\tau}$, to build the scheme,

$$
\bar{v}_{j+l}=\frac{1}{\Delta x_{j+l}} \int_{I_{j+l}} G(x) d x, \quad l=-2,-1,0,1,2,
$$


in which the linear weights, $c_{0}, c_{1}, c_{2}$, are expressed by

$$
\begin{aligned}
& c_{0}=\frac{\frac{1}{30}-\frac{\rho_{j}\left(x_{0}^{(n)}\right)^{2}}{8}+\frac{\rho_{j}\left(x_{0}^{(n)}\right)^{4}}{24}}{\frac{1}{3}-\rho_{j}\left(x_{0}^{(n)}\right)+\frac{\rho_{j}\left(x_{0}^{(n)}\right)^{2}}{2}}, \\
& c_{1}=\frac{-\frac{1}{30}+\frac{4 \rho_{j}\left(x_{0}^{(n)}\right)}{45}+\frac{9 \rho_{j}\left(x_{0}^{(n)}\right)^{2}}{80}-\frac{13 \rho_{j}\left(x_{0}^{(n)}\right)^{3}}{36}+\frac{11 \rho_{j}\left(x_{0}^{(n)}\right)^{4}}{144}+\frac{\rho_{j}\left(x_{0}^{(n)}\right)^{5}}{8}-\frac{\rho_{j}\left(x_{0}^{(n)}\right)^{6}}{24}}{\left(\frac{1}{3}-\rho_{j}\left(x_{0}^{(n)}\right)+\frac{\rho_{j}\left(x_{0}^{(n)}\right)^{2}}{2}\right)\left(-\frac{1}{6}+\frac{\rho_{j}\left(x_{0}^{(n)}\right)^{2}}{2}\right)}, \\
& c_{2}=\frac{-\frac{1}{20}+\frac{\rho_{j}\left(x_{0}^{(n)}\right)}{12}+\frac{\rho_{j}\left(x_{0}^{(n)}\right)^{2}}{8}-\frac{\rho_{j}\left(x_{0}^{(n)}\right)^{3}}{6}+\frac{\rho_{j}\left(x_{0}^{(n)}\right)^{4}}{24}}{-\frac{1}{6}+\frac{\rho_{j}\left(x_{0}^{(n)}\right)^{2}}{2}} .
\end{aligned}
$$

3.2.2 $g^{\prime}(x, t)<0$ in the SL-FV scheme

Third-order WENO reconstruction The identical arithmetic is applied to create this condition, so, simply, in the templates $S_{\tau}=\bigcup_{l=0}^{1} I_{j+1+\tau-l}, \tau=0,1$, we design the polynomials

$$
H_{\tau}(x)=\operatorname{COE}_{1}\left(\frac{1}{2}-\rho_{j}(x)\right)+\operatorname{COE}_{2} \quad(\tau=0,1)
$$

as well as

$$
\bar{v}_{j+\tau-l}=\frac{1}{\Delta x_{j+\tau-l}} \int_{I_{j+\tau-l}}\left(\operatorname{COE}_{1}\left(\frac{1}{2}-\rho_{j}(x)\right)+C O E_{2}\right) d x, \quad \tau=0,1, l=0,1,
$$

so we can acquire the multinomials

$$
\begin{aligned}
& H_{1}\left(x_{0}^{(n)}\right)=\left(\frac{1}{2}-\rho_{j}\left(x_{0}^{(n)}\right)\right) \bar{v}_{j+1}^{n}+\left(\frac{1}{2}+\rho_{j}\left(x_{0}^{(n)}\right)\right) \bar{v}_{j}^{n}, \\
& H_{2}\left(x_{0}^{(n)}\right)=-\left(\frac{1}{2}+\rho_{j}\left(x_{0}^{(n)}\right)\right) \bar{v}_{j+2}^{n}+\left(\frac{3}{2}+\rho_{j}\left(x_{0}^{(n)}\right)\right) \bar{v}_{j+1}^{n},
\end{aligned}
$$

and we use the template $S=\bigcup_{\tau=0}^{1} S_{\tau}$ to establish the multinomial

$$
G(x)=\operatorname{COE}_{1}\left(\frac{1}{2}-\rho_{j}(x)\right)^{2}+\operatorname{COE}_{2}\left(\frac{1}{2}-\rho_{j}(x)\right)+\mathrm{COE}_{3},
$$

as well as

$$
\bar{v}_{j+l}=\frac{1}{\Delta x_{j+l}} \int_{I_{j+l}}\left(\operatorname{COE}_{1}\left(\frac{1}{2}-\rho_{j}(x)\right)^{2}+\operatorname{COE}_{2}\left(\frac{1}{2}-\rho_{j}(x)\right)+\operatorname{COE}_{3}\right) d x, \quad l=0,1,2,
$$

so we can acquire the multinomial

$$
\begin{aligned}
G\left(x_{0}^{(n)}\right)= & \left(-\frac{1}{6}+\frac{\rho_{j}\left(x_{0}^{(n)}\right)^{2}}{2}\right) \bar{v}_{j+2}^{n}+\left(\frac{5}{6}-\rho_{j}\left(x_{0}^{(n)}\right)-\rho_{j}\left(x_{0}^{(n)}\right)^{2}\right) \bar{v}_{j+1}^{n} \\
& +\left(\frac{1}{3}+\rho_{j}\left(x_{0}^{(n)}\right)+\frac{\rho_{j}\left(x_{0}^{(n)}\right)^{2}}{2}\right) \bar{v}_{j}^{n},
\end{aligned}
$$


and we can find the linear weights, expressed by $c_{0}, c_{1}$,

$$
c_{0}=\frac{\frac{1}{3}+\rho_{j}\left(x_{0}^{(n)}\right)+\frac{\rho_{j}\left(x_{0}^{(n)}\right)^{2}}{2}}{\frac{1}{2}+\rho_{j}\left(x_{0}^{(n)}\right)}, \quad c_{1}=-\frac{-\frac{1}{6}+\frac{\rho_{j}\left(x_{0}^{(n)}\right)^{2}}{2}}{\frac{1}{2}+\rho_{j}\left(x_{0}^{(n)}\right)} .
$$

We chose the following form as the smoothness indicators in the stencils $S_{0}, S_{1}$ :

$$
I Y_{0}=\left(\bar{v}_{j+1}^{n}-\bar{v}_{j}^{n}\right)^{2}, \quad I Y_{1}=\left(\bar{v}_{j+2}^{n}-\bar{v}_{j+1}^{n}\right)^{2} .
$$

The corresponding nonlinear weights can be written as

$$
\omega_{\tau}=\frac{\delta_{\tau}}{\sum_{s=1}^{2} \delta_{s}}, \quad \delta_{\tau}=\frac{c_{\tau}}{\left(\varepsilon+I Y_{\tau}\right)^{2}}, \quad \tau=0,1
$$

Here $\varepsilon>0$ is to keep the denominator away from 0 , and $\varepsilon$ is utilized as $10^{-6}$ for the simulations.

Thus, we have the reconstructed point value of $v$ at $x_{0}^{(n)}$ at time level $t^{n}$, which can be indicated by

$$
\left(v_{0}^{(n)}\right)^{-}=\sum_{\tau=0}^{1} \omega_{\tau} H_{\tau}\left(x_{0}^{(n)}\right) .
$$

Fifth-order WENO reconstruction The identical arithmetic is used to build this condition, so, simply, we use the templates $S_{\tau}=\bigcup_{l=0}^{2} I_{j+1+\tau-l}, \tau=0,1,2$, and $S=\bigcup_{\tau=0}^{2} S_{\tau}$ to establish the fifth-order scheme in this case.

\subsection{SL-FV WENO schemes for the Euler equations}

We begin to study the Euler equation in this section, which is given by

$$
\mathbf{v}_{t}+\mathbf{g}(\mathbf{v})_{x}=0,
$$

where

$$
\mathbf{v}=\left(\begin{array}{c}
\rho \\
\rho u \\
E
\end{array}\right), \quad \mathbf{g}(\mathbf{v})=\left(\begin{array}{c}
\rho u \\
\rho u^{2}+p \\
u(E+p)
\end{array}\right) .
$$

In the above equation, the density is $\rho, u$ is the velocity, $p$ is the pressure, and $E$ is the total energy. The total energy is given as

$$
E=\frac{p}{\gamma-1}+\frac{1}{2} \rho u^{2},
$$

with $\gamma=1.4$.

Parallel to the scalar case, we can readily acquire the approach for the Euler equation (3.3) as follows: the scheme is got by resolving the integrated version of (3.3) in space and time,

$$
\overline{\mathbf{v}}_{j}^{n+1}=\overline{\mathbf{v}}_{j}^{n}-\frac{1}{\Delta x_{j}} \int_{t^{n}}^{t^{n+1}}\left(\hat{\mathbf{g}}_{j+\frac{1}{2}}-\hat{\mathbf{g}}_{j-\frac{1}{2}}\right) d t,
$$


in which $\hat{\mathbf{g}}$ is a numerical flux. Identical to deducing of the scheme (3.2), the SL-FV scheme for the Euler equation (3.3) is obtained by

$$
\overline{\mathbf{v}}_{j}^{n+1}=\overline{\mathbf{v}}_{j}^{n}-\frac{1}{\Delta x_{j}} \sum_{l=0}^{m-1} B(l)\left(\mathbf{D}_{l}\left(v\left(x_{0}^{(n)}, t^{n}\right)\right)-\mathbf{D}_{l}\left(v\left(x_{j-\frac{1}{2}}^{(n)}, t^{n}\right)\right)\right) .
$$

Here, $l$ is also even number and $\mathbf{D}_{l}(v(x, t))=\frac{\partial^{l} \hat{\mathbf{g}}(v(x, t))}{\partial t^{l}}$, the approximations $\mathbf{v}\left(x_{0}^{(n)}, t^{n}\right)$ and $\mathbf{v}\left(x_{j-\frac{1}{2}}^{(n)}, t^{n}\right)$ need to be reconstructed. As we know, the Euler system has three eigenvalues, namely

$$
\varphi_{1}(\mathbf{v}), \quad \varphi_{2}(\mathbf{v}), \quad \varphi_{3}(\mathbf{v})
$$

Li et al. [27] use them to decouple the equation to follow the curves to find the point $x_{0}^{(n)}$ truly, the method is also taken in our article. The SL-FV WENO schemes of the Euler system adopt the same WENO schemes as used in the scalar conservation laws. However, different from the scalar case, the conditions $\varphi_{i} \geq 0$ and $\varphi_{i}<0$ are considered in the Euler system.

In contrast to the scalar case, we need to adopt a typical local characteristic decomposition procedure to achieve non-oscillatory performance, which is briefly described as follows:

- At the point $x_{j+\frac{1}{2}}$, compute the average value for given cell averages $\mathbf{v}_{j}$.

$$
\tilde{\mathbf{v}}_{j+\frac{1}{2}}=\frac{1}{2}\left(\mathbf{v}_{j}+\mathbf{v}_{j+1}\right)
$$

- Compute the Jacobian matrix $\mathbf{g}^{\prime}\left(\tilde{\mathbf{v}}_{j+\frac{1}{2}}\right)$ denoted by $\mathbf{A}_{j+\frac{1}{2}}$, the $m \times m$ matrix $\mathbf{R}_{j+\frac{1}{2}}\left(\tilde{\mathbf{v}}_{j+\frac{1}{2}}\right)$ composed of its right eigenvectors, and the $m \times m$ matrix $\mathbf{R}_{j+\frac{1}{2}}^{-1}\left(\tilde{\mathbf{v}}_{j+\frac{1}{2}}\right)$ composed of its left eigenvectors

$$
\mathbf{A}_{j+\frac{1}{2}}=\mathbf{g}^{\prime}\left(\tilde{\mathbf{v}}_{j+\frac{1}{2}}\right), \quad \mathbf{R}_{j+\frac{1}{2}}=\mathbf{R}_{j+\frac{1}{2}}\left(\tilde{\mathbf{v}}_{j+\frac{1}{2}}\right), \quad \mathbf{R}_{j+\frac{1}{2}}^{-1}=\mathbf{R}_{j+\frac{1}{2}}^{-1}\left(\tilde{\mathbf{v}}_{j+\frac{1}{2}}\right) .
$$

- For each target cell $I_{j}$, project $\mathbf{v}_{j}$ to the characteristic field locally by using the transformation

$$
\mathbf{u}_{i}=\mathbf{R}_{j+\frac{1}{2}}^{-1} \mathbf{v}_{i}
$$

where $i=j-2, \ldots, j+3$ for the fifth-order WENO reconstructions.

- Perform scalar reconstructions on each component of $\mathbf{u}$ to get $\mathbf{u}_{j+\frac{1}{2}}^{ \pm}$.

- $\mathbf{v}_{j+\frac{1}{2}}^{ \pm}=\mathbf{R}_{j+\frac{1}{2}} \mathbf{u}_{j+\frac{1}{2}}^{ \pm}$.

The local characteristic decomposition procedure is now well defined.

Remark 3.1 Note that when the linear weights $c_{0}, c_{1}, c_{2}$, and $c_{3}$ are negative, the approach will become one of instability. Shi et al. [28] propose a method, which is used to tackle the negative weights, which involves few cost and is quite effective. The method can keep the scheme with negative weights steady and achieve high-order precision. 


\section{Numerical results}

In this article, the mathematical simulations of third-order SL-FV and fifth-order SL-FV processes are provided for scalars and Euler equations. In our experiments, the CFL condition is bound to be 1.9 in the linear scalar equations, 2.9 in the nonlinear scalar conservation law, and 9.9 in the Euler systems. Uniform meshes are used in all simulations. The boundary conditions of all the examples are periodic in this paper.

\subsection{The scalar problems}

Example 1 (The linear conservation law) In this example, the linear scalar conservation law has the following form:

$$
v_{t}+v_{x}=0
$$

The starting condition is $v(x, 0)=\sin (x)$, the interval $[0,2 \pi]$ is the computational region. We have the equation

$$
v(x, t)=\sin (x-t)
$$

which is a precise result in this linear scalar equation. The final time is $t=5$, and CFL $=1.9$. The errors and orders of this problem are displayed in Table 1. The SL-FV WENO processes can achieve third-order and fifth-order accuracy. From the table, we can understand that the SL-FV WENO processes maintain the planned orders of preciseness.

Example 2 (The nonlinear conservation law) In this example, we study the equation below, which is called the Burgers equation,

$$
v_{t}+\left(\frac{v^{2}}{2}\right)_{x}=0
$$

$v(x, 0)=0.5+\sin (x)$ is a starting condition of this problem. The computational region is $[0,2 \pi]$. The final time is $t=0.3$ and $C F L=2.9$. Table 2 lists their errors and orders about the $L^{1}$ and $L^{\infty}$ norms. Significantly, the order of the SL-FV WENO approaches can reach third- and fifth-order accuracy, respectively. So we can easily observe that the SL-FV WENO approaches maintain the planned orders of preciseness. We continue to discuss this example, the solution is computed up to $t=1.5$ when a shock has already appeared in the result. In Figure 1, we show the results of the FV-SL WENO approaches with $N=50$. According to this, we can see that the high-order methods give non-oscillatory shock transitions in this example.

\begin{tabular}{|c|c|c|c|c|c|c|c|c|}
\hline \multirow[t]{2}{*}{$N$} & \multicolumn{4}{|l|}{ SL-FV 3} & \multicolumn{4}{|l|}{ SL-FV 5} \\
\hline & $L^{1}$ error & Order & $L^{\infty}$ error & Order & $L^{1}$ error & Order & $L^{\infty}$ error & Order \\
\hline 20 & $9.04 \mathrm{E}-04$ & - & $1.41 \mathrm{E}-03$ & - & $1.58 \mathrm{E}-04$ & - & $2.46 \mathrm{E}-04$ & - \\
\hline 40 & $1.41 \mathrm{E}-04$ & 2.68 & $2.22 \mathrm{E}-04$ & 2.67 & $5.05 \mathrm{E}-06$ & 4.97 & 7.92E-06 & 4.96 \\
\hline 80 & $1.64 \mathrm{E}-05$ & 3.10 & $2.58 \mathrm{E}-05$ & 3.11 & $1.58 \mathrm{E}-07$ & 5.00 & $2.49 \mathrm{E}-07$ & 4.99 \\
\hline 160 & $2.09 \mathrm{E}-06$ & 2.97 & $3.29 \mathrm{E}-06$ & 2.97 & $5.31 \mathrm{E}-09$ & 4.90 & $8.35 \mathrm{E}-09$ & 4.90 \\
\hline 320 & $2.62 \mathrm{E}-07$ & 2.99 & $4.11 \mathrm{E}-07$ & 3.00 & $1.63 \mathrm{E}-10$ & 5.03 & $2.05 \mathrm{E}-10$ & 5.06 \\
\hline
\end{tabular}

Table 1 The semi-Lagrangian finite volume WENO 3 and WENO 5 methods on Example 1 with $t=5, \mathrm{CFL}=1.9$ 
Table 2 The semi-Lagrangian finite volume WENO 3 and WENO 5 methods on Example 2 with $t=0.3, \mathrm{CFL}=2.9$

\begin{tabular}{|c|c|c|c|c|c|c|c|c|}
\hline \multirow[t]{2}{*}{$N$} & \multicolumn{4}{|l|}{ SL-FV 3} & \multicolumn{4}{|l|}{ SL-FV 5} \\
\hline & $L^{1}$ error & Order & $L^{\infty}$ error & Order & $L^{1}$ error & Order & $L^{\infty}$ error & Order \\
\hline 20 & $1.07 \mathrm{E}-03$ & - & $3.40 \mathrm{E}-03$ & - & $1.15 \mathrm{E}-04$ & - & $5.22 \mathrm{E}-04$ & - \\
\hline 40 & $1.30 \mathrm{E}-04$ & 3.04 & $4.47 \mathrm{E}-04$ & 2.93 & $4.05 \mathrm{E}-06$ & 4.83 & $2.08 \mathrm{E}-05$ & 4.65 \\
\hline 80 & $1.60 \mathrm{E}-05$ & 3.02 & $5.84 \mathrm{E}-05$ & 2.94 & $1.27 \mathrm{E}-07$ & 5.00 & $7.21 \mathrm{E}-07$ & 4.85 \\
\hline 160 & $1.98 \mathrm{E}-06$ & 3.01 & $7.19 \mathrm{E}-06$ & 3.02 & $3.96 \mathrm{E}-09$ & 5.00 & $2.31 \mathrm{E}-08$ & 4.96 \\
\hline 200 & $1.01 \mathrm{E}-06$ & 3.02 & $3.67 \mathrm{E}-06$ & 3.01 & 1.30E-09 & 4.99 & $7.58 \mathrm{E}-09$ & 4.99 \\
\hline
\end{tabular}

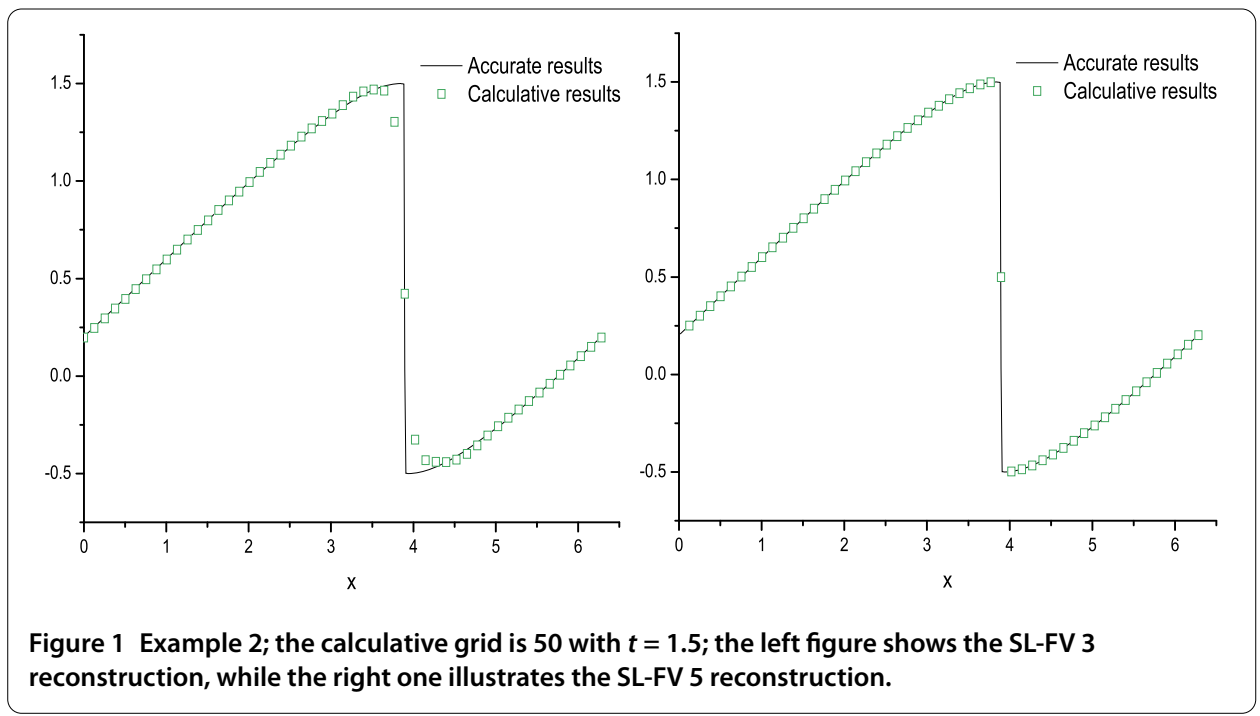

Table 3 The semi-Lagrangian finite volume WENO 3 and WENO 5 methods on Example 3 with $t=2, \mathrm{CFL}=9.9$

\begin{tabular}{|c|c|c|c|c|c|c|c|c|}
\hline \multirow[t]{2}{*}{$N$} & \multicolumn{4}{|l|}{ SL-FV 3} & \multicolumn{4}{|l|}{ SL-FV 5} \\
\hline & $\overline{L^{1} \text { error }}$ & Order & $L^{\infty}$ error & Order & $L^{1}$ error & Order & $L^{\infty}$ error & Order \\
\hline 20 & $1.91 \mathrm{E}-03$ & - & $2.99 \mathrm{E}-03$ & - & $4.01 \mathrm{E}-05$ & - & $6.22 \mathrm{E}-05$ & - \\
\hline 40 & $2.54 \mathrm{E}-04$ & 2.91 & $3.98 \mathrm{E}-04$ & 2.91 & $1.27 \mathrm{E}-06$ & 4.98 & $1.99 \mathrm{E}-06$ & 4.97 \\
\hline 80 & $3.22 \mathrm{E}-05$ & 2.98 & $5.05 \mathrm{E}-05$ & 2.98 & 4.10E-08 & 4.95 & $6.15 \mathrm{E}-08$ & 5.02 \\
\hline 160 & $4.03 \mathrm{E}-06$ & 3.00 & $6.33 \mathrm{E}-06$ & 3.00 & $1.29 \mathrm{E}-09$ & 4.99 & $1.84 \mathrm{E}-09$ & 5.06 \\
\hline 200 & $2.07 \mathrm{E}-06$ & 2.99 & $3.24 \mathrm{E}-06$ & 3.00 & $4.24 \mathrm{E}-10$ & 4.99 & $5.73 \mathrm{E}-10$ & 5.23 \\
\hline
\end{tabular}

\subsection{Euler systems}

Example 3 In the problem, the orders of the SL-FV WENO methods for Euler system are tested. We consider (3.3) with starting condition $\rho(x, 0)=1+0.2 \sin (x), u_{0}=p_{0}=1$. In this example, the computational region is $[0,2]$. The time stop is at $t=2$ and $\mathrm{CFL}=9.9$. The precise result of the one-dimensional Euler system is $\rho(x, t)=1+0.2 \sin (x-t)$, $u(x, t)=p(x, t)=1$. Table 3 describes the situation of the errors and orders about the $L^{1}$ and $L^{\infty}$ norms. We have third-order and fifth-order accuracy in this example. From this table, we can point out that the designed orders of the SL-FV WENO methods are accomplished. 

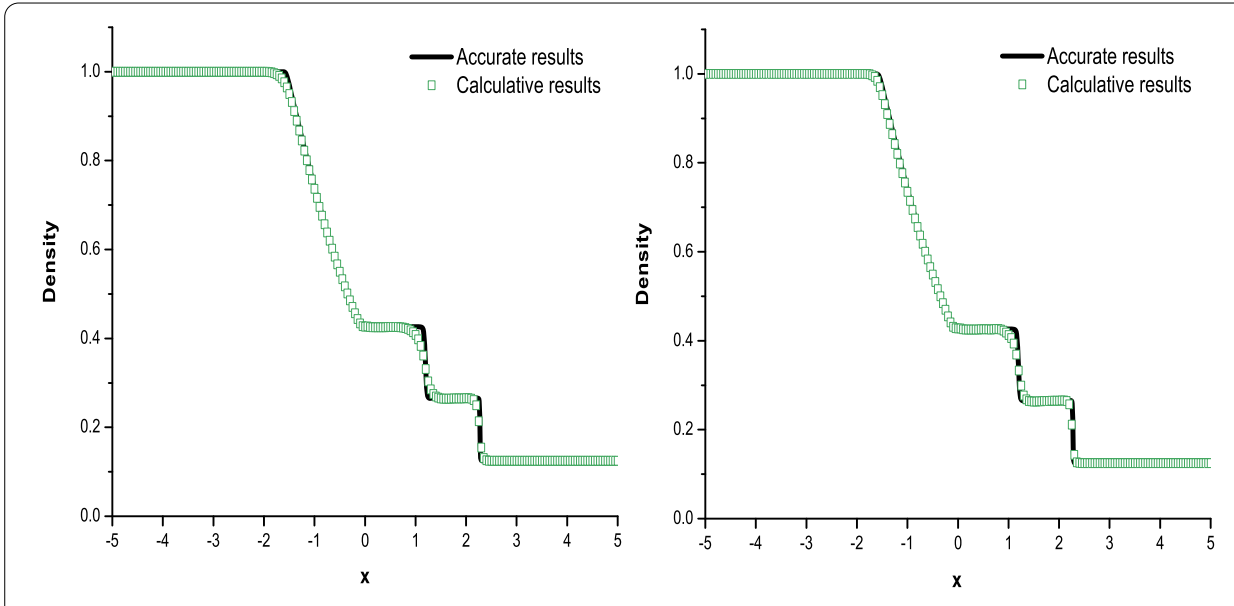

Figure 2 Example 4; the calculative grid is 200; the left figure shows the SL-FV 3 reconstruction, while the right one illustrates the SL-FV 5 reconstruction.

Example 4 (The Sod problem) In the example, the Sod problem is studied, the following starting condition is adopted in (3.3):

$$
(\rho, u, p)= \begin{cases}(1,0,1), & x \leq 0, \\ (0.125,0,0.1), & x>0 .\end{cases}
$$

We consider $[-5,5]$ as the investigative region. The resolutions are up to $t=1.3$ with $\mathrm{CFL}=$ 9.9. We apply 200 cells and the semi-Lagrangian finite volume WENO methods to plot the solutions. In Figure 2, we display the plots for the density computed by the high-order SL-FV methods, though the numerical outcomes show that the SL-FV WENO methods can reach sharpness without spurious oscillations.

Example 5 (The Lax problem) The example with the coming initial condition in the equation (3.3) is studied

$$
(\rho, u, p)= \begin{cases}(0.445,0.698,3.528), & x \leq 0 \\ (0.5,0,0.571), & x>0 .\end{cases}
$$

We consider the computational region of the example $[-5,5]$. We utilize the semiLagrangian finite volume WENO 3 method and WENO 5 method to compute the results, the time stop at $t=1.3$ with $\mathrm{CFL}=9.9$. In Figure 3, we exhibit the plots for density are computed by the SL-FV WENO 3 and WENO 5 methods, respectively. The figure demonstrates that there are no shocks and it captures the profile of the wave interaction well.

Example 6 (The stationary contact discontinuity problem) The stationary contact discontinuity problem with the following starting condition, which is the Euler equation (3.3)

$$
(\rho, u, p)= \begin{cases}(1.4,0,1), & x \leq 0 \\ (1,0,1), & x>0\end{cases}
$$



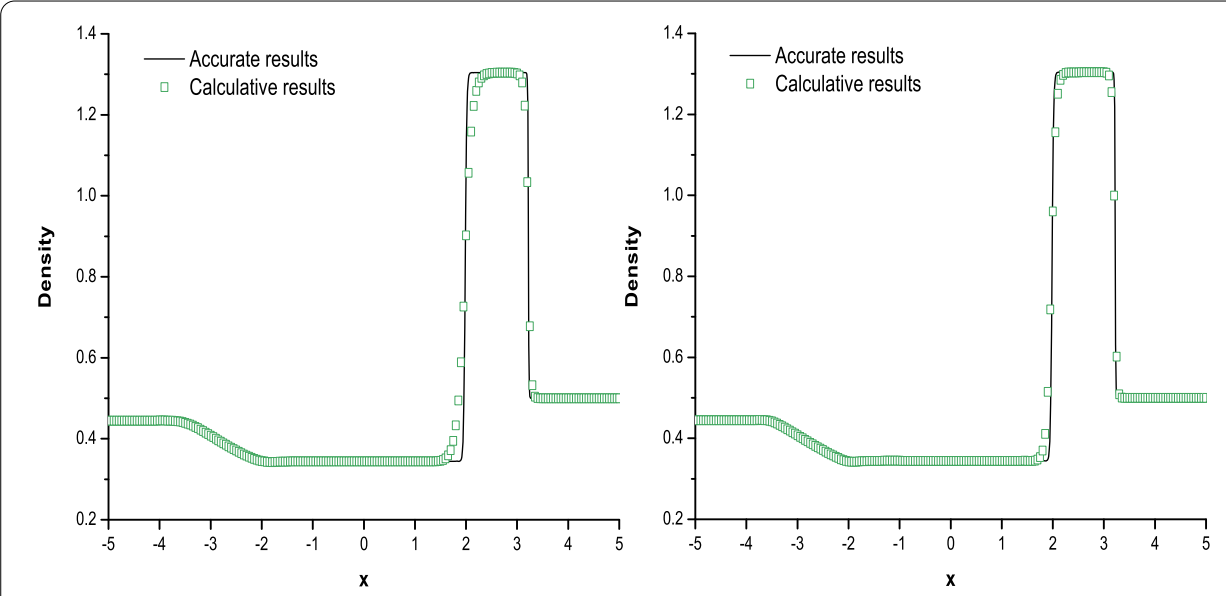

Figure 3 Example 5; the calculative grid is 200; the left figure shows the SL-FV 3 reconstruction, while the right one illustrates the SL-FV 5 reconstruction.
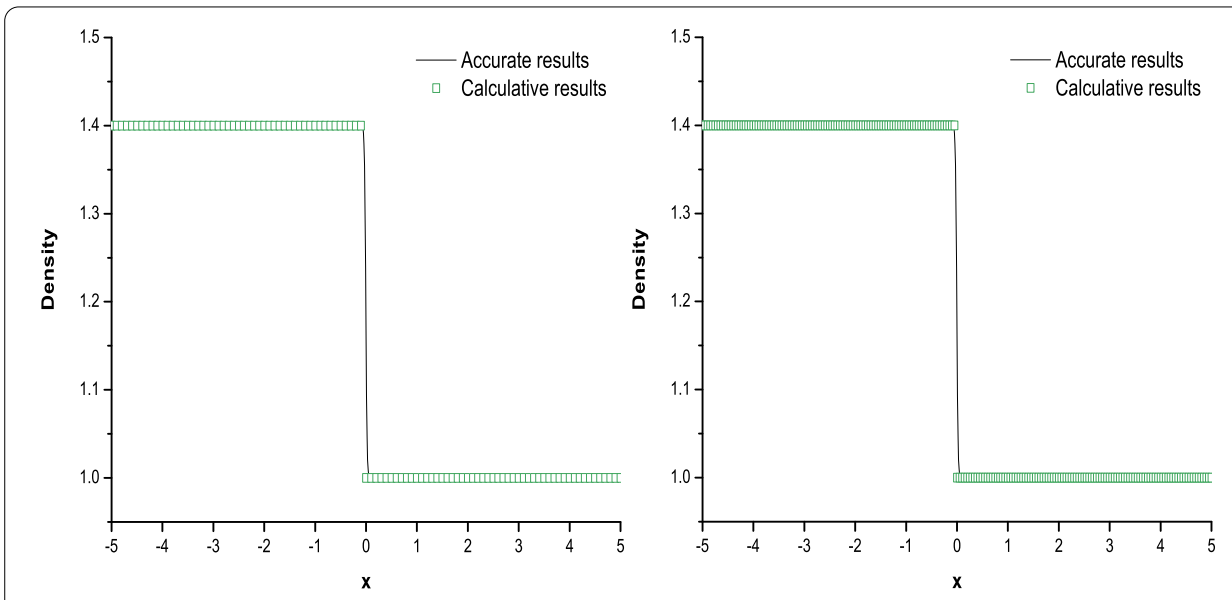

Figure 4 Example 6; the SL-FV 3 reconstruction; the left figure is with 100 calculative grids, while the right one is with 200 calculative grids.

We consider the computational region of this problem $[-5,5]$. The results up to $t=1.3$ with $\mathrm{CFL}=9.9$. The outcomes of the third-order SL finite volume reconstruction are exhibited in Figure 4, which contains a mesh of 100 points and 200 points. From the figure, we can point out that the computational results are reasonable and the method can capture discontinuities well.

Example 7 (The tiny density and internal energy problem) The tiny density and internal energy problem is considered in this example, which is the Euler equation (3.3), and the starting condition can be written as

$$
(\rho, u, p)= \begin{cases}(1,-2,0.4), & x \leq 0, \\ (1,2,0.4), & x>0 .\end{cases}
$$

We consider the computational region of this problem $[-5,5]$. The results are up to $t=1.3$ with $\mathrm{CFL}=9.9$. We utilize the SL-FV WENO 3 process to plot the solutions on mesh of 

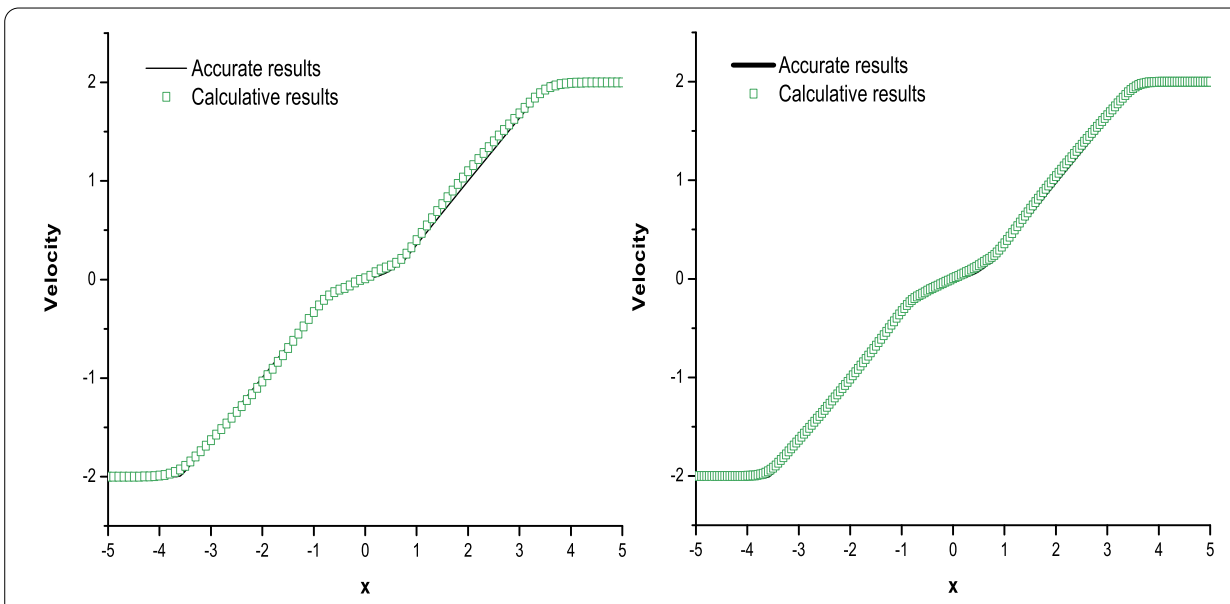

Figure 5 Example 7; the SL-FV 3 reconstruction; the left figure is with 100 calculative grids, while the right one is with 200 calculative grids.
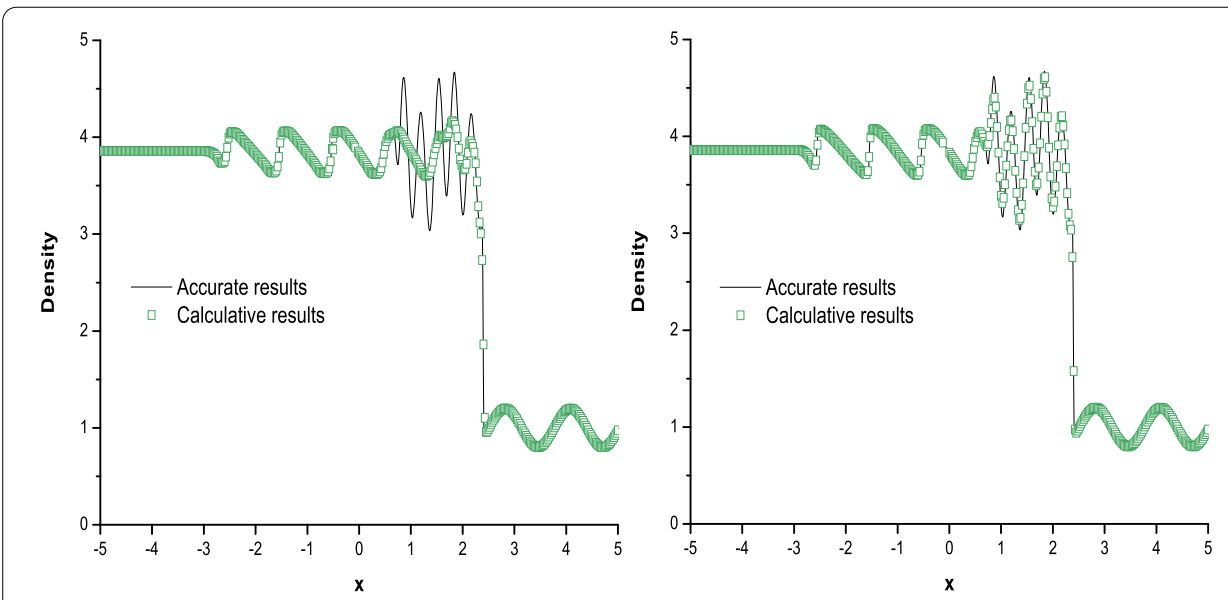

Figure 6 Example 8; the calculative grid is 400; the left figure shows the SL-FV 3 reconstruction, while the right one illustrates the SL-FV 5 reconstruction.

100 points and 200 points. In Figure 5, we present the plots of the velocity reconstructed by the WENO 3 process. The figure reveals that the computational results of the proposed method in this paper are satisfactory.

Example 8 (The shock density wave interaction problem) This example adopts the following starting condition in the equation (3.3), which can be written as

$$
(\rho, u, p)= \begin{cases}(3.857143,2.629369,10.333333), & x \leq-4 \\ (1+0.2 \sin 5 x, 0,1), & x>-4 .\end{cases}
$$

We investigate the computational region of the example $[-5,5]$. The results are up to $t=1.8$ with $\mathrm{CFL}=9.9$. In Figure 6, the numerical results of the semi-Lagrangian on a uniform mesh are exhibited, which contain third-order and fifth-order reconstructions. From the figure, we can understand that the SL-FV WENO 5 process is more accurate than the 

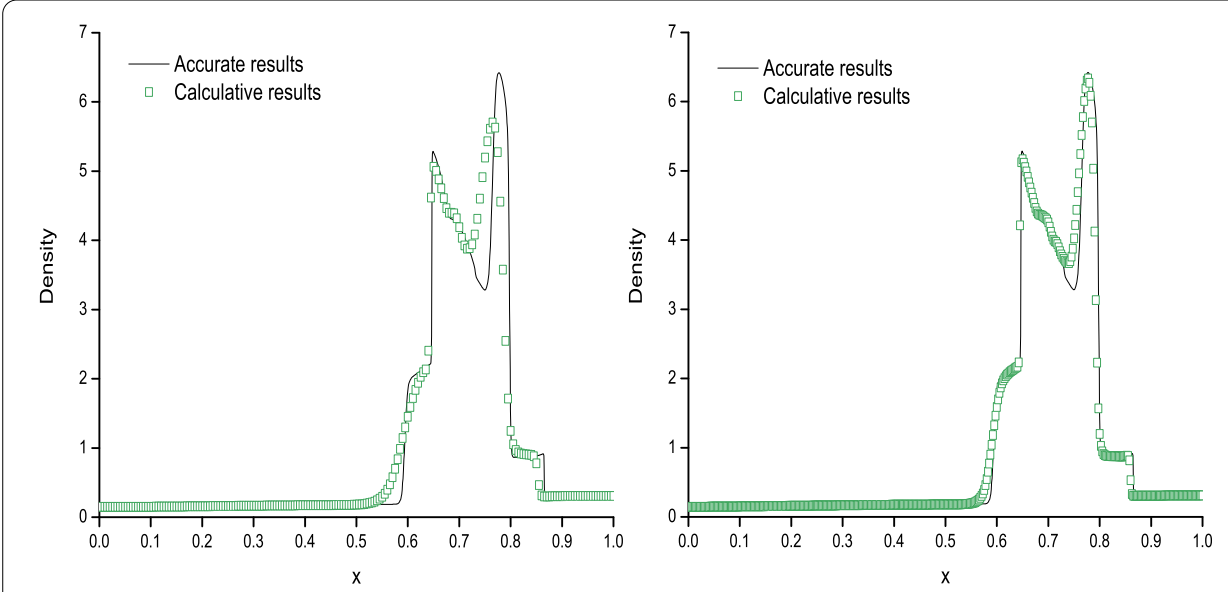

Figure 7 Example 9; the SL-FV 5 reconstruction; the left figure is with 200 calculative grids, while the right one is with 400 calculative grids.

SL-FV WENO 3 reconstruction and they can capture the profile of the wave interaction well.

Example 9 (The interacting blast waves problem) This example is the Euler equation (3.3) and adopts the following starting condition, which can be written as

$$
(\rho, u, p)= \begin{cases}(1,0,1000), & 0 \leq x \leq 0.1 \\ (1,0,0.01), & 0.1 \leq x \leq 1, \\ (1,0,100), & \text { otherwise. }\end{cases}
$$

We consider the computational region of the example is $[0,1]$. The results stop at $t=0.038$ with $\mathrm{CFL}=9.9$. In Figure 7, we exhibit the plots for variables that are computed by the fifth-order SL-FV method with a uniform grid of 200 points and 400 points, respectively. From the figure, we can point out that the computational results are reasonable and the method can capture the discontinuities well.

\section{Concluding remarks}

The high-order SL-FV approaches presented above simulate the results of the scalar and Euler equations in this paper. The designed method, without restriction about the CFL time step, maintains uniform high-order precision in both space and time. Many onedimensional experiments are performed based on SL-FV WENO 3 and WENO 5 reconstructions in order to validate the performance and efficiency of the proposed methods for scalar and conservation law systems. Further details regarding the construction of highorder SL-FV methods for multidimensional equations are still under dispute, and these are the primary direction of future research. 


\section{Author details}

${ }^{1}$ Department of Mathematics, Harbin Institute of Technology, No. 92, West Da-Zhi Street, Harbin, 150001, China. ${ }^{2}$ School of Management, Harbin Institute of Technology, No. 92, West Da-Zhi Street, Harbin, 150001, China.

\section{Acknowledgements}

This work is supported by the National Science Foundation of China $(11271100,11301113,71303067)$, Harbin Science and Technology Innovative Talents Project of Special Fund (2013RFXYJ044), China Postdoctoral Science Foundation funded project (Grant No. 2013M541400), the Heilongjiang Postdoctoral Fund (Grant No. LBH-Z12102), the Fundamental Research Funds for the Central Universities (Grant No. HIT.HSS.201201).

\section{Received: 5 September 2014 Accepted: 26 December 2014 Published online: 21 March 2015}

\section{References}

1. Cheng, J, Shu, C-W: A third order conservative Lagrangian type scheme on curvilinear meshes for the compressible Euler equations. Commun. Comput. Phys. 4, 1008-1024 (2008)

2. Mohammadian, A, Le Roux, D: Conservative semi-implicit semi-Lagrangian scheme for simulation of shallow flows. Comput. Phys. Commun. 174, 99-108 (2006)

3. Restelli, M, Bonaventura, L, Sacco, R: A semi-Lagrangian discontinuous Galerkin method for scalar advection by incompressible flows. J. Comput. Phys. 216, 195-215 (2006)

4. Wang, B, Zhao, G, Fringer, O: Reconstruction of vector fields for semi-Lagrangian advection on unstructured staggered grids. Ocean Model. 40, 52-71 (2011)

5. Luo, H, Baum, J, Löhner, R: On the computation of multi-material flows using ALE formulation. J. Comput. Phys. 194 304-328 (2004)

6. Cheng, J, Shu, C-W: A high order ENO conservative Lagrangian type scheme for the compressible Euler equations. J. Comput. Phys. 227, 1567-1596 (2007)

7. Liu, W, Cheng, J, Shu, C-W: High order conservative Lagrangian schemes with Lax-Wendroff type time discretization for the compressible Euler equations. J. Comput. Phys. 228, 8872-8891 (2009)

8. Cheng, J, Shu, C-W: A cell-centered Lagrangian scheme with the preservation of symmetry and conservation proprieties for compressible fluid flows in two-dimensional cylindrical geometry. J. Comput. Phys. 229, 7191-7206 (2010)

9. Cheng, J, Shu, C-W: A Lagrangian scheme with the preservation of symmetry and conservation in cylindrical geometry: preliminary study. Proc. Comput. Sci. 1, 1903-1911 (2010)

10. Cheng, C, Knorr, G: The integration of the Vlasov equation in configuration space. J. Comput. Phys. 22, $330-351$ (1976)

11. Crouseilles, N, Respaud, T, Sonnendrucker, E: A forward semi-Lagrangian method for the numerical solution of the Vlasov equation. Comput. Phys. Commun. 180, 1730-1745 (2009)

12. Crouseilles, N, Mehrenberger, M, Sonnendrucker, E: Conservative semi-Lagrangian schemes for Vlasov equation. J. Comput. Phys. 229, 1927-1953 (2010)

13. Acar, R: Oscillation-free advection of interfaces with high order semi-Lagrangian schemes. Comput. Fluids 38 137-159 (2009)

14. Nair, R, Scroggs, J, Semazzi, F: A forward-trajectory global semi-Lagrangian transport scheme. J. Comput. Phys. 190 275-294 (2003)

15. White, J, Dongarra, J: High-performance high-resolution semi-Lagrangian tracer transport on a sphere. J. Comput. Phys. 230, 6778-6799 (2011)

16. Qiu, J-M, Shu, C-W: Positivity preserving semi-Lagrangian discontinuous Galerkin formulation. J. Comput. Phys. 230, 8386-8409 (2011)

17. Qiu, J-M, Christlieb, A: A conservative high order semi-Lagrangian WENO method for the Vlasov equation. J. Comput. Phys. 229, 1130-1149 (2010)

18. Qiu, J-M, Shu, C-W: Conservative high order semi-Lagrangian finite difference WENO methods for advection in incompressible flow. J. Comput. Phys. 230, 863-889 (2011)

19. Qiu, J-M, Shu, C-W: Conservative semi-Lagrangian finite difference WENO formulations with applications to the Vlasov equation. Commun. Comput. Phys. 10, 979-1000 (2011)

20. Rossmanith, J, Seal, D: A positivity-preserving high-order semi-Lagrangian discontinuous Galerkin scheme for the Vlasov-Poisson equations. J. Comput. Phys. 230, 6203-6232 (2011)

21. Liu, X, Osher, S, Chan, T: Weighted essentially non-oscillatory schemes. J. Comput. Phys. 115, $200-212$ (1994)

22. Jiang, G, Shu, C-W: Efficient implementation of weighted ENO schemes. J. Comput. Phys. 126, $202-228$ (1996)

23. Montranal, P, Shu, C-W: Real gas computation using an energy relaxation method and high-order WENO schemes. J. Comput. Phys. 148, 59-80 (1999)

24. Shu, C-W, Osher, S: Efficient implementation of essentially non-oscillatory shock-capturing schemes. J. Comput. Phys. 77, 439-471 (1988)

25. Zhu, J, Qiu, J, Liu, T, Khoo, BC: RKDG methods with WENO type limiters and conservative interfacial procedure for one-dimensional compressible multi-medium flow simulations. Appl. Numer. Math. 61, 554-580 (2011)

26. Shu, C-W: Essentially non-oscillatory and weighted essentially non-oscillatory schemes for hyperbolic conservation laws. In: Quarteroni, A (ed.) Cockburn, B, Johnson, C, Shu, C-W, Tadmor, E, Advanced Numerical Approximation of Nonlinear Hyperbolic Equations. Lecture Notes in Math., vol. 1697, pp. 325-432. Springer, Berlin (1998)

27. Ii, S, Xiao, F: CIP/multi-moment finite volume method for Euler equations: a semi-Lagrangian characteristic formulation. J. Comput. Phys. 222, 849-871 (2007)

28. Shi, J, Hu, C, Shu, C-W: A technique of treating negative weights in WENO schemes. J. Comput. Phys. 175, 108-127 (2002) 